\title{
Rechtsextreme Diskurse im Unterricht analysieren. Eine theoriegeleitete Medienanalyse mit Schüler/innen
}

\author{
*karin.golser@stud.sbg.ac.at, Holztechnikum Kuchl \\ **thomas.jekel@sbg.ac.at, Fachbereich für Geographie und Geologie \& School of Education, Universität Salzburg ( $₫$ korresp. Autor) \\ eingereicht am: 08.03.2018, akzeptiert am: 14.07.2018
}

In ganz Europa und darüber hinaus entwickelte sich in den letzten Jahren eine völlig neue (und sehr alte) Diskursstruktur, die im Wesentlichen als das Wiedererstarken rechten/rechtsextremen Gedankenguts aufgefasst werden kann. Dazu gibt es sowohl alltagsweltliche, als auch naturgemäß viele wissenschaftliche Analysen. Dieser Beitrag stellt eine im Unterricht getestete Methodik vor, die versucht, Diskurse der ,neuen Rechtsextremen` auch im Unterricht mit Schüler/innen forschend zu rekonstruieren. Dieser geht vom Syndromansatz sowie von der Idee semantischer Kategorien und Topoi aus, stellt Unterrichtsmaterialien vor und beschreibt die Ergebnisse eines ersten Unterrichtseinsatzes.

Keywords: Rechtsextremismus; Diskursanalyse; Politische Bildung

\section{Analyzing rightwing-extremism in secondary education: A theory-based media-analysis with students}

In Europe and beyond, the 'new right' has gained significant importance in recent times, changing discourses both in nationalist and neo-liberal ways. This has been analysed in public media as well as academia. This contribution presents a model and materials that allow reconstructing right-wing discourses in a research based learning scenario in a short secondary school research project. It is based on the 'syndrome approach' of right-wing extremism theory as well as the idea of semantic categories and topoi. Finally, it discusses first teaching experiences from an Austrian school with pupils aged 17-18 years.

Keywords: rightwing extremism; discourse analysis; citizenship education

\section{$1 \quad$ Einleitung}

Spätestens seit dem Jahr 2015 geht mit der vermeintlichen ,Flüchtlingskrise' ein deutlicher politischer als auch gesellschaftlicher Rechtsruck in Europa einher. Rechte und rechtsextreme Parteien, wie der ,Front National' in Frankreich, die ,AfD' in Deutschland, die ,FPÖ‘ in Österreich, ,Fidesz' in Ungarn oder ,PiS‘ in Polen, um nur einige wenige Beispiele zu nennen, gewinnen zunehmend an Bedeutung und Zuspruch. Erste Vorboten in Richtung eines politischen und gesellschaftlichen Rechtsrucks in Österreich brachte die Präsidentschaftswahl im Jahr 2016, wo es zu einer Stichwahl zwischen dem FPÖ-Kandidaten Norbert Hofer und dem grünen Kandidaten Alexander Van der Bellen kam. Hier wurden die Grenzen des ,Sagbaren' im Wahlkampf deutlich verschoben (vgl. Fuchs 2016). Spätestens seit der Nationalratswahl 2017, wo ein ,Anti-Ausländer-Wahlkampf vorherrschte, der sich häufig gegen Flüchtlinge und nicht EU-Bürger/ innen richtete, kann eine klare politische Bewegung nach rechts identifiziert werden. Zudem gibt es neben politischen Parteien eine Vielzahl von rechten europäischen Bewegungen, die sich gegen Nicht-Europäer/ innen bzw. gegen Migrant/innen wenden, wie etwa die Bewegung, Patriotische Europäer gegen die Islamisierung des Abendlandes' (kurz,PEGIDA') die ,Identitären', die ,Alt.Rightbewegung' in den USA oder die ,Blood \& Honour-Bewegung', die enormen Popularitätszuwachs verzeichnen. Es werden also beispielsweise vermeintliche Unterschiede zwischen ,Europäer/ innen' und ,Nicht-Europäer/innen' sowie zwischen ,Weißen' und ,Farbigen' usw. konstruiert, die sowohl der „ideologischen, politischen und/oder praktischen Diskriminierung auf allen gesellschaftlichen Ebenen [dienen]" (Wodak 2012: 425). Denn solange daran geglaubt wird, dass die Mitglieder einer Gesellschaft Homogenität aufweisen, gibt es genügend Bemühungen, sich von ,anderen ' abzugrenzen bzw. die scheinbaren eigenen Werte zu verteidigen (vgl. Pelinka 2013). 
Vor allem neue soziale Medien werden aktuell vermehrt von rechten und rechtsextremen Bewegungen als Verbreitungskanäle für die xenophobe Stimmungsmache in der Gesellschaft genutzt, gleichzeitig die Mainstream-Medien von rechten Bewegungen relativ pauschal als linkslastig charakterisiert. Gerade die sozialen Medien erlauben dabei, weitgehend unkontrolliert, ,alternative Weltsichten' und , alternative Facts' $\mathrm{zu}$ verbreiten. Rassistische Meinungen, Stereotype sowie Vorurteile werden geschaffen und durch ihre mediale Verbreitung in gewisser Weise auch legitimiert (vgl. Wodak 2012). Soziale Medien beziehungsweise Online-Medien mit Kommentarfunktion ermöglichen der Bürgerin/dem Bürger eine Teilhabe am Diskurs. Häufig bedienen sich die Beiträge aus der rechten Szene einer stark vereinfachenden und generalisierenden sowie einer metaphorisch fremdenfeindlichen Sprache. Dies bedeutet, dass viele Äußerungen von einem hetzerischen und unreflektierten Charakter geprägt sind und den Inhalten der Rechtstaatlichkeit widersprechen, wobei vor allem Menschenrechte gemäß der Universal Declaration of Human Rights (United Nations 1948) verletzt werden. Festmachen kann man dies am ,Straftatbestand der Verhetzung', zu finden unter $\$ 283$ im Strafgesetzbuch mit folgendem Wortlaut: „einer feindseligen Handlung gegen eine im Inland bestehende Kirche oder Religionsgesellschaft oder gegen eine durch ihre Zugehörigkeit zu einer solchen Kirche oder Religionsgesellschaft, zu einer Rasse, zu einem Volk, einem Volksstamm oder einem Staat bestimmte Gruppe auffordert oder aufreizt " beziehungsweise öffentlich gegen eine Gruppe von Menschen „hetzt, sie beschimpft oder verächtlich zu machen sucht" (BMJ 2017). In den vergangenen Jahren ist in Österreich der Straftatbestand der Verhetzung immer öfter angezeigt worden. Dennoch klagen die Staatsanwaltschaften selten an. Als weiteres Indiz für ein Anwachsen rechter Bewegungen können die Zahlen von rechtsextremen Straftaten herangezogen werden: Diese haben sich zwischen dem Jahr 2005 und 2015 mehr als verfünffacht (vgl. Mernyi \& Bauer 2017).

Vor allem Schüler/innen sind Nutzer/innen von (neuen) Medien und kommen daher mit den medial verbreiteten Diskursen in Berührung. Ziel der vorgestellten Unterrichtssequenz ist es, die Schüler/innen im Umgang mit Medien und Sprache zu sensibilisieren, sodass sie mediale Inhalte kritisch hinterfragen und ihre vermeintliche ,Objektivität' nicht als gegeben hinnehmen, sondern als ,Scheinobjektivität ' entlarven. Das entworfene Unterrichtsmaterial ermöglicht Schülerinnen und Schülern, sich mit verschiedenen Onlinemedien auseinanderzusetzen. Die Schüler/innen werden innerhalb der Unterrichtsumgebung mit semantischen Strategien, die im Kontext von rechtsextremen Diskursen anzutreffen sind, konfrontiert und in einem weiteren
Schritt mithilfe einer theoriegeleiteten Medienanalyse dazu befähigt, Rechtsextremismus zu identifizieren. ${ }^{1}$

Außerdem soll gezeigt werden, dass sich die Behandlung von rechtsextremen Diskursen gerade im Fach Geographie und Wirtschaftskunde besonders gut eignet - vor allem wenn es darum geht, den Anspruch des Faches, nämlich mehrperspektivisch und politisch bildend wirksam zu sein, zu erfüllen (vgl. Bundesministerium für Bildung 2016). Zudem sind überdurchschnittlich viele der Argumentationsstrukturen der rechten Szene explizit räumlich (vgl. Golser \& Jekel 2017).

\section{Kennzeichen rechtsextremer Diskurse ${ }^{2}$}

Um rechtsextreme Diskurse in adäquater Form identifizieren zu können, werden Holzers, Syndromphänomene' als auch Zapata-Barreros und Rubio-Carboneros (2014) semantische Kategorien und Topoi als theoretische Grundlagen herangezogen. Folgend werden die zentralen Inhalte sowie mögliche Gemeinsamkeiten beider Ansätze kurz vorgestellt.

Bei Holzers ,Syndromphänomenen ' (1994) handelt es sich um ein Bündel von miteinander verbundenen Merkmalen bzw. Prinzipien, durch das Rechtsextremismus gekennzeichnet ist:

- Das Prinzip ,Natur' oder ,Natürlichkeit‘: Wird eine Person beispielsweise aus ethnischen Gründen abgelehnt, so erlaubt es dieses Prinzip, sie als, widernatürlich' abzuwerten. Es entsteht eine Ideologie der sozialen und globalen Ungleichheit und damit verbundene Hierarchien werden legitimiert (vgl. Holzer 1994, Bailer-Galanda o.J.; Fuchs 2016; Wodak 2012; Jekel et al. 2017).

- Das Prinzip ,Volk und Volksgemeinschaft': Bestärkt die Ansicht, dass ein Volk/eine Volksgemeinschaft mit unveränderlichen Charaktereigenschaften ausgestattet sei, die dem Individuum teilweise sogar übergeordnet sind. Daraus resultiert die Nichtigkeit der einzelnen Person; lediglich ihre Pflichten bestimmen die Position in der Gesellschaft (vgl. Holzer 1994).

- Ethnozentrismus und -pluralismus': Es kommt

Es muss explizit darauf verwiesen werden, dass sich die Autor/ innen der Existenz einer linksextremen Szene bewusst sind. Es ist aber anzumerken, dass unter der Annahme eines Syndromansatzes nach Holzer (1994) sowie der Suche nach semantischen Kategorien und Topoi nach Zapata-Barrero \& Rubio-Carbonero (2014) dieses Analyseinstrument für jede soziale Bewegung neu zu entwickeln und zu kalibrieren wäre.

2 Grundsätzlich bieten sich hier als theoretische Grundlagen die Extremismustheorie (z. B. Jaschke 1991; 1994) sowie der Syndromansatz (Holzer 1994) an. Wegen des spezifischen Bezugs auf genau rechte soziale Bewegungen wird hier auf den Syndromansatz, der Merkmalsgruppen in den Vordergrund stellt, und nicht auf die Extremismustheorie, die sich auf antidemokratischen Gehalt sowie eine ,Distanz zur gesellschaftlichen Mitte bezieht, zurückgegriffen. 
zu einem latenten und zur Selbstüberhöhung neigendem ,Wir-Gefühl' und alle nicht dazugehörenden Menschen / Gruppen werden ausgegrenzt. Jede Durchmischung von ,Gruppen 'wird als Bedrohung der ,Eigengruppe verstanden. Mögliche Visionen wären ,Deutschland den Deutschen', sowie die ,Türkei den Türken' etc. (vgl. Holzer 1994; Bailer-Galanda o. J.) Dieses Element von Rechtsextremismus kann zum System weltweiter Apartheid führen, da es ein ähnlich exklusives bzw. inklusives Potenzial wie der Rassismus der 1930er Jahre aufweist (vgl. Bailer-Galanda o.J.). Auch Fuchs (2016) manifestiert eine engmaschige Verbindung zwischen Rassismus und Nationalismus, allerdings seien es aktuell eher Unterscheidungen kultureller und politischer statt ehemals biologischer bzw. ethnischer Natur, die zur Inklusion bzw. Exklusion von Gruppen führen.

- Die ,Sündenbockfunktion: Einzelne Gruppen, wie beispielsweise ,die Ausländer', ,die Politiker', ,die Wissenschaftler' usw. werden für Missstände jeglicher Art verantwortlich gemacht (vgl. Holzer 1994). Eine besondere Dynamik entsteht, wenn Menschen ihre sozialen bzw. ökonomischen Ängste auf vermeintlich dafür verantwortliche Feindgruppen projizieren. Außerdem entsteht so eine Entlastungs- bzw. Integrationsfunktion nach innen. Verschwörungstheorien sind oftmals das Ergebnis, denen größtenteils mehr Beachtung und Glauben geschenkt wird als rationalen Analysen (vgl. Bailer-Galanda o. J.).

- Nationalisierende Geschichtsbetrachtung‘: Bestehen Brüche in der historischen Entwicklung einer Nation, die beispielsweise ihre Würde gefährden, so kommt es zu keinem kritisch-reflexiven Umgang, sondern zu kecken Neuschöpfungen sowie zu Verschleierungen der Wahrheit. Als ein ,Paradebeispiel' kann das NS-Regime herangezogen werden, wo ein Spannungsfeld zwischen Schuld und Glorifizierung der Verbrechen, die im Namen des ,deutschen Volkes' begangen wurden, identifizierbar ist und es nach wie vor zu Verharmlosungen sowie zu Verleugnungen kommt.

Als ein weiterer theoretischer Zugang, um Rechtsextremismus zu identifizieren beziehungsweise Elemente von rechtsextremer Ideologie aufzuzeigen, können semantische Kategorien und Topoi nach Zapata-Barrero \& Rubio-Carbonero (2014) herangezogen werden. Es handelt sich dabei sowohl um sprachliche als auch um thematische ,Stilmittel', die häufig in recht(sextrem)en Diskursen mit der Absicht, Hass und Ablehnung gegen Gruppen bzw. Individuen zu generieren, anzutreffen sind. Wesentliche semantische Kategorien sind hierbei:
- Verwendung von negativen Generalisierungen: eine Methode, um Diskriminierung und Ausgrenzung zu erzeugen. Ein negatives Verhalten bzw. negative Charaktermerkmale eines Individuums werden auf eine gesamte soziale Gruppe übertragen und dies führt daher zu generellen Vereinfachungen, sprich zu ,Typenbeschränkungen', die keine Ausnahmen zulassen. Sowohl negative Verallgemeinerungen als auch Vereinfachungen sind die Basis für Stereotype und Vorurteile (vgl. ebd.).

- Verwendung von negativen ,Hyperbeln': Generalisierungen werden dramatisiert und übertrieben. Negative ,Übertreibungen' sind zumeist Bestandteil von politischen Diskursen und für Migrations- und Ausländerdebatten, wie beispielsweise durch Vorhersagen von nationalen Krisen oder bürgerkriegsartigen Zuständen, geradezu prädestiniert (vgl. ebd.).

- Verwendung von negativen Metaphern: Sie werden sowohl für die Beschreibung von Objekten, als auch von Subjekten gebraucht. Eine gut durchdachte Metapher, die für den Rezipienten/die Rezipientin gut nachvollziehbar ist, kann durchaus zu einer veränderten Wahrnehmung der Realität führen (vgl. ebd.). Außerdem kann sie durch Pointierungen oder Verschleierungen von Inhalten einen beachtlichen Einfluss darauf haben, wie Realität verstanden wird. Im Kontext von Rechtsextremismus werden Metaphern oftmals verwendet, um Angst und Ablehnung gegen Menschen/ Gruppen zu schüren (vgl. ebd.).

- Verwendung von ,naturalisierter Sprache': Es handelt sich um eine ,objektivierende Sprache', die bis zur ,Entmenschlichung' reichen kann (vgl. Jekel \& Huber 2005; Holzer 1994). Innerhalb der Bevölkerung können oftmals negative Gefühle, wie beispielsweise Angst identifiziert werden. Als mögliche Reaktion darauf erfolgt keine Konfrontation mit den Ängsten, sondern es kommt zur pauschalen Ablehnung des ,Fremden'. Eine damit verbundene populäre und auf allen Ebenen der Gesellschaft ,zulässige' Strategie ist, ein gemeinsames ,Wir' zu erzeugen, um sich von der, out-group', die eine augenscheinliche ,Andersartigkeit' aufweist, abzugrenzen (vgl. Zapata-Barrero \& Rubio-Carbonero 2014; van Houtum \& van Naerssen 2002).

Die vier genannten semantischen Kategorien, die in der Rhetorik von Rechtspopulistinnen und -populisten und Rechtsextremistinnen und -extremisten besonders präsent sind, generieren weitere argumentative Instrumente (Topoi), die gegen ,feindliche Gruppen eingesetzt werden können. Beispiele hierfür sind die Topoi der Bedrohung, der Belastung sowie der Bevorteilung. Im vorliegenden Kontext basieren Topoi auf 
einem ,angeblich' gemeinsamen Glauben, welcher einerseits den ,gesunden Menschenverstand' anspricht und andererseits als eine ,nicht-hinterfragbare Wahrheit' etabliert wird und somit keine Rechtfertigung benötigt (vgl. Zapata-Barrero \& Rubio-Carbonero 2014). Ein Topos entsteht daher aus einem unreflektierten Prozess und kann als eine Art sozialer Glaube, der in einem konkreten Kontext auf Werte und Normen beruht, definiert werden (vgl. ebd.).

Es kann festgestellt werden, dass die vorgestellten Kategorien und Topoi einen eindeutigen Raumbezug aufweisen. Allen semantischen Kategorien wohnen Alteritätskonstruktionen, sprich Konstruktionen von einem gemeinsamen, Wir' gegen ein ,Nicht-wir', inne. Es handelt sich um direkte räumliche Zugänge, wie beispielsweise um Lebensräume und dadurch identifizierbare Charaktermerkmale verschiedener Gruppen, die zu ,bordering'-, , ordening- und ,othering-Prozessen führen, um sich von ,Fremden' bzw. ,Anderen' abzugrenzen (vgl. Pichler 2017; Jekel et al. 2017; van Houtum \& van Naerssen 2002).

\section{Unterrichtskonzeption}

Für die Unterrichtskonzeption wurden sowohl Golser \& Jekel (2017) als auch Jekel, Lehner \& Vogler (2017) herangezogen. Beide Artikel versuchen, Rechtsextremismus zu definieren und diesen in einem weiteren Schritt auf unterschiedliche Weise mit Schüler/innen zu erörtern. Jekel et al. (2017) verfolgen mit ihrer Initiative \#notmysymbol' einen kritisch-aktivistischen Zugang, welcher sich auf eine gemeinsame Kartierung von rechtsextremer Symbolik, die im öffentlichen Raum trotz Verbotsgesetz häufig anzutreffen ist, konzentriert. Golser und Jekel (2017) stützen sich hingegen auf eine semantische Analyse eines kartographischen Mediums. Die rechtsextremen Inhalte der ,Einzelfallkarte', eine von Laien erstellte Onlinekarte, die explizit darauf abzielt, mutmaßlich verübte ,kriminelle Delikte von Ausländer/innen aufzuzeigen, wird anhand von Holzers (1994) sowie Zapata-Barreros \& Rubio-Carboneros (2014) Prinzipien sowie semantischen Kategorien und Themen dekonstruiert.

Der vorliegende Unterrichtsentwurf wurde, wenn auch etwas vereinfacht, entlang des didaktischen Vorschlags zur Dekonstruktion der ,Einzelfallkarte' konzipiert. In einem ersten Schritt wird eine Analyse der Rezipientinnen und Rezipienten bzw. der Autorenschaft der Karte sowie der dahinterliegenden Daten und Fakten empfohlen. In einem weiteren Schritt sollen mögliche rechtsextreme Inhalte der Karte durch eine theoriegeleitete Textanalyse, welche sich auf Holzer (1994) sowie auf Zapata-Barrero \& Rubio-Carbonero (2014) stützt, identifiziert werden (vgl. Golser \& Jekel 2017).

\subsection{Struktur}

Die Unterrichtsumgebung ermöglicht den Schülerinnen und Schülern selbständig eine vorab getroffene Auswahl von medialen Inhalten zu durchforsten und rechtsextreme Strategien zu identifizieren. Als Zeitfenster sind mindestens zwei bis maximal drei Unterrichtseinheiten angedacht. Idealerweise handelt es sich bei den ersten beiden Unterrichtseinheiten um eine Doppeleinheit, sodass die Schüler/innen so flexibel wie möglich arbeiten können. Die Unterrichtsumgebung wurde in Anlehnung an das KIOSK-Modell von Teml \& Teml (2006: 9f.) erstellt, welche die Phasen „Kontakt, Information, Organisation, selbstständiges Lernen und Kontrolle" unterscheiden. Darüber hinaus kommen durchmischte Sozialformen zum Einsatz, welche einen schüler/innen-zentrierten Unterricht gewähren. Durch Partner- und Gruppenarbeiten erlernen sie den Erwerb von fachlichem Wissen, Selbständigkeit sowie das Arbeiten im Team und die Bereitschaft zur Kooperation, sodass sowohl Interaktions- als auch Handlungskompetenzen ausgebaut werden (vgl. Meyer 2011). Konkret hinterfragen die Schüler/innen bestehende Normen, Aussagen und Meinungen, wodurch nach Vielhaber (1999) ein kritisches gesellschaftlich-emanzipatorisches Potenzial identifiziert werden kann.

\subsection{Lehrplanbezug}

Aufgrund der Komplexität der Thematik, die vor allem durch die sprachliche Anforderung gegeben ist, ist es nahezu unumgänglich, die Unterrichtsumgebung in der Sekundarstufe II anzusiedeln. Zieht man den semestrierten Lehrplan des Bundesministeriums für Bildung (2016) für die AHS heran, so ergeben sich Anknüpfungspunkte für folgende Klassen bzw. Semester:

- 5. Klasse (1. und 2. Semester): Bevölkerung und Gesellschaft diskutieren

- 5. Klasse (3. Semester, Kompetenzmodul 3): Konvergenzen und Divergenzen europäischer Gesellschaften erörtern

- 7. Klasse (6. Semester, Kompetenzmodul 6): Demographische Entwicklung und gesellschaftspolitische Implikationen beurteilen

Neben der klassischen Verortung nach Schulstufen bzw. Semestern können zudem die Basiskonzepte als Orientierungshilfen, Andockmöglichkeiten und Relevanzfilter herangezogen werden (vgl. Fridrich 2016; Jekel \& Pichler 2017). In der vorliegenden Unterrichtsumgebung wird versucht, den Schülerinnen und Schülern multiperspektivische Zugänge anzubieten. Es wird daher das Basiskonzept der Kontin- 
genz berücksichtigt, welches die „Thematisierung von Mehrdeutigkeiten unterrichtsrelevanter Phänomene aus den Bereichen Gesellschaft, Politik, Ökonomie und Ökologie, aber auch den Blick auf vielfältige Lösungsansätze" verlangt (Bundesministerium für Bildung 2016).

Zudem wird das Basiskonzept ,Interessen, Macht und Konflikt', welches die Aufmerksamkeit auf soziale, ökonomische und ökologische Abhängigkeiten, Konflikte und Krisen lenkt, berücksichtigt (vgl. ebd.). Außerdem wird der Frage nach Inklusions- und Exklusionsprozessen hinlänglich der Beteiligung an Entscheidungsprozessen nachgegangen. „Die Schüler/ innen sollen sich über die Gewinner/innen und Verlierer/innen von politischen Entscheidungsprozessen klar werden und sich selbst als Akteure/Akteurinnen in Aushandlungsprozessen erleben können" (ebd.).

Neben den Basiskonzepten für das Fach Geographie und Wirtschaftskunde soll an dieser Stelle auch auf das Unterrichtsprinzip ,Politische Bildung (BMBWF 2015) verwiesen werden. Denn dieser Grundsatzerlass ermöglicht es, die vorliegende Unterrichtsumgebung in mehreren Unterrichtsfächern durchzuführen. Abseits von Geographie und Wirtschaftskunde wäre die Unterrichtsumgebung sowohl im Unterrichtsfach Geschichte, Sozialkunde und Politische Bildung als auch in Deutsch durchführbar. Das Ziel des Unterrichtsprinzips ,Politische Bildung' ist, Schüler/innen zu selbständigen Urteilen und sozialem Verständnis zu führen. Dazu gehört das Erkennen fehlender Trennschärfe von wissenschaftlichen Definitionen, das Erfahren der Schwierigkeiten von Textkategorisierungen und das Kennenlernen sehr unterschiedlicher, aber in sich konsistenter Weltentwürfe, die in dieser Unterrichtsumgebung anhand des Phänomens ,Rechtsextremismus' diskutiert werden.

„Die Lebens- und Erfahrungswelt der Schüler/innen, die aktuell durch Prozesse der Globalisierung, Phänomene der Mediendemokratie und zunehmend nicht-nationalstaatliche oder gar mehrfachen ,Identitäten 'von Bürger/innen geprägt ist, gilt es im Unterricht verstärkt miteinzubeziehen" (ebd.). Die Politische Bildung soll daher „einen aktiven Beitrag zur Gestaltung der Gesellschaft sowie zur Verwirklichung und Weiterentwicklung der Demokratie und Menschenrechte" leisten (ebd.). Auf diese beiden Forderungen des Unterrichtsprinzips der Politischen Bildung wird durch die Datengrundlage (sozialer Geo-/ Medien) sowie durch den Auszug aus der Menschenrechtskonvention Bezug genommen.

\subsection{Kommentierter Ablauf}

Die Schüler/innen werden zunächst mit dem Begriff ,Rechtsextremismus' konfrontiert und erhalten die
Aufgabe, weitere Begriffe, die sie damit assoziieren, aufzuschreiben (AA1). Wichtig ist, dass die Assoziationen in Einzelarbeit notiert werden. Danach werden die Notizen der Schüler/innen durch die Lehrperson abgesammelt. Ziel ist es, durch einen aktivierenden Unterrichtseinstieg erste Präkonzepte der Schüler/innen zu erheben.

In einem zweiten Schritt wird versucht, ein gemeinsames Verständnis des Begriffes Rechtsextremismus zu entwickeln. Dazu erhalten die Schüler/innen ein Methodenblatt, auf dem zunächst mögliche Definitionen von (Rechts-)Populismus in Abgrenzung von Rechtsextremismus präsentiert werden. Die Schüler/innen machen sich erste Gedanken über die Trennschärfe von Rechtsextremismus und -populismus (AA2). Zudem findet sich ein Auszug zu Menschenrechten, der einerseits ihre Grundbotschaft in verkürzter Form wiedergibt und andererseits die Verfassungswidrigkeit von rechtsextremen Handlungen unterstreicht.

Danach werden die Schüler/innen mit semantischen Kategorien, Themen und Prinzipien nach Holzer (1994) und Zapata-Barrero \& Rubio-Carbonero (2014), die in Verbindung mit Rechtsextremismus häufig gebraucht werden, konfrontiert. Es folgt ein Arbeitsauftrag, bei dem die Definitionen der entwickelten Kategorien mit Beispielen angeführt sind. In einem weiteren Schritt versuchen die Schüler/innen Überschriften aus ,aktuellen' Zeitungsartikeln, sowohl aus Print- als auch aus Onlinemedien, den verschiedenen Kategorien zuzuordnen (AA3). Das Ziel dieser Aufgabe ist zu zeigen, dass die Überschriften nicht immer eindeutig zuordenbar sind und daher auch mehrere Möglichkeiten zulässig sind.

Nach der Zuordnungsübung, die das nötige Handwerkszeug liefert, gehen die Schüler/innen in die Phase der Gruppenarbeit. Gearbeitet wird entlang von vorgegebenen Pfaden, die aus Onlinezeitungsartikeln sowie Kommentaren der Leser/innen und einer Onlinekarte bestehen (AA4). Ist Zeit übrig, so scheint es empfehlenswert, die Hoax-Map (www.hoaxmap.org) als ein Beispiel für sauber recherchierte Informationen und als, Countermap' anzusprechen.

Um zu sehen, ob sich das Verständnis von Rechtsextremismus unmittelbar nach den Einheiten verändert hat, werden erneut anonyme Assoziationen zum Begriff gesammelt (AA5).

In einer weiteren Unterrichtseinheit ist es empfehlenswert, die Ergebnisse der Schüler/innen zu besprechen. Zur Visualisierung der Assoziationen eigenen sich, Word Clouds' besonders gut. Jene Begriffe, die in der Visualisierung größer erscheinen, wurden öfter genannt und sind daher von größerer subjektiver Bedeutung. Auch die Ergebnisse aus den Gruppenarbeiten sollten entsprechend diskutiert und resümiert werden. 


\subsection{Planungsraster}

\begin{tabular}{|c|c|c|c|}
\hline Ablauf & Inhalt & Sozialform & Zeit \\
\hline Einstieg & Kurze Begrüßung & Plenum & \\
\hline $\begin{array}{l}\text { Kontakt } \\
\text { Ziel = Abrufen von Präkonzepten! }\end{array}$ & $\begin{array}{l}\mathrm{AA}^{3} 1 \text { : Brainstorming zum Begriff, Rechtsextremismus'. } \\
\text { S/S schreiben Begriffe auf, die sie damit assoziieren. } \\
\text { S/S arbeiten anonymisiert in Einzelarbeit. Ausschließlich } \\
\text { Begriffe \& absammeln durch die Lehrperson. }\end{array}$ & Einzelarbeit & $10^{\prime}$ \\
\hline $\begin{array}{l}\text { Information } \\
\text { Ziel = Entwicklung eines Verständnisses! }\end{array}$ & $\begin{array}{l}\text { AA2: Methodenblatt, Rechtspopulismus/Rechtsextremismus } \\
\text { und Auszug zu Menschenrechten' } \\
\text { AA3: Zuordnung Überschriften zu sprachlichen Kategorien, } \\
\text { Themen und Prinzipien. }\end{array}$ & $\begin{array}{l}\text { Einzelarbeit } \\
\text { Partnerarbeit }\end{array}$ & $30^{\prime}$ \\
\hline PAUSE/Organisation & EINTEILUNG Gruppen, Arbeitsaufträge via Klassenverteiler (Mail). & & $5^{\prime}$ \\
\hline $\begin{array}{l}\text { Selbstständiges Lernen, } \\
\text { selbständiges Erarbeiten } \\
\text { Ziel= identifizieren der verschiedenen Kate- } \\
\text { gorien, die innerhalb der Medien verwendet } \\
\text { werden! }\end{array}$ & $\begin{array}{l}\text { AA4: S/S arbeiten in Gruppen von max. } 4 \text { Personen } \\
\text { (Gruppen doppelt besetzen): } \\
\text { - Gruppe 1: Artikel \& verlinkte Leserbriefe/ Postings. } \\
\text { Überschrift: "Künftig nur } 560 € \text { Sozialhilfe für Migranten" } \\
\text { - Gruppe 24: Artikel mit Kommentaren. Überschrift: } \\
\text { "Leben mit Zuwanderern: Stimmung verschlechtert" } \\
\text { - Gruppe 35: Einzelfallkarte mit dem Fokus auf Salzburg } \\
\text { - Gruppe 4: Einzelfallkarte mit dem Fokus auf Wien }\end{array}$ & $\begin{array}{l}\text { Gruppenarbeit } \\
\text { Aufgaben via Mail } \\
\text { via Mail an Lehrperson } \\
\text { zurück - es wird alles im } \\
\text { Dokument bearbeitet! }\end{array}$ & $35^{\prime}$ \\
\hline $\begin{array}{l}\text { Anregung Reflexion } \\
\text { Ziel = als ein Beispiel, wie sauber recher- } \\
\text { chierte Informationen aussehen - Wider- } \\
\text { legung von Falschaussagen! }\end{array}$ & $\begin{array}{l}\text { Hoaxmap: } \\
\text { siehe unter: www.hoaxmap.org }\end{array}$ & Plenum & $10^{\prime}$ \\
\hline $\begin{array}{l}\text { Abschlussreflexion } \\
\text { Ziel = geschärfte, weitere Konzepte von } \\
\text { Rechtsextremismus! }\end{array}$ & $\begin{array}{l}\text { AA5: Arbeitsauftrag: Brainstorming zum Thema } \\
\text {,Rechtsextremismus' } \\
\text { Ausschließlich Begriffe und erneutes absammeln. }\end{array}$ & Einzelarbeit & $10^{\prime}$ \\
\hline $\begin{array}{l}\text { Kontrolle } \\
\text { Ziel = verändertes Verständnis? }\end{array}$ & $\begin{array}{l}\text { Besprechung offener Fragen \& Ergebnispräsentation: } \\
\text { Brainstorming durch Visualisierung der Word Clouds }\end{array}$ & Plenum & $15-20^{\prime}$ \\
\hline
\end{tabular}

AA steht für Arbeitsauftrag.

4 wird im Punkt "Arbeitsaufgaben" und unter "Ergebnisse der Schüler/innen und Erfahrungen" exemplarisch dargestellt.

5 wird im Punkt "Arbeitsaufgaben" exemplarisch dargestellt. 


\subsection{Materialien}

\section{- Assoziationen zum Begriff ,Rechtsextremismus' - Durchgang 1 / Durchgang 2}

AA1/AA5: Notiere alles, was dir zu diesem Begriff einfällt/was du mit diesem Begriff verbindest.

\section{- Methodenblatt Rechtspopulismus/Rechtsextremismus in (neuen) Medien ${ }^{6}$}

AA2: Lies den kurzen Auszug über Populismus und überlege, ob es scharfe Grenzen zwischen Rechtspopulismus und Rechtsextremismus gibt.

\section{Populismus:}

Populismus betont häufig den Gegensatz zwischen dem ,Volk' und der ,Elite' und nimmt dabei in Anspruch, auf der Seite des, einfachen Volkes' zu stehen. Populismus hat kein bestimmtes eigenes Wertesystem. Populismus kann daher mit ganz unterschiedlichen politischen Richtungen und Zielsetzungen einhergehen, wobei er oft zur ,Stimmungsmache' für oder gegen etwas eingesetzł wird. Oftmals wenden sich Politiker/innen, Protestparteien, aber auch soziale Bewegungen mithilfe von Populismus an die Öffentlichkeit, um eine größere Wirkung zu erzielen.

\section{Populismus und Medien:}

Politisches Handeln beziehungsweise politische Entscheidungen werden nahezu ausschließlich über mediale Berichterstattungen an die ,Gesellschaft' weitergegeben. Populistische Ansichten werden über zahlreiche mediale Kanäle, die von Boulevardzeitungen bis über soziale Medien reichen, verbreitet. Es gilt zu bedenken, dass man mit der Presseberichterstattung nicht nur über Politik informiert, sondern auch Politik machen kann.

\section{Rechtspopulismus:}

Allgemein wird häufig über die Begriffe ,Linkspopulismus' und ,Rechtspopulismus' diskutiert, wobei sich viele Themen überschneiden können. Anfang des 21 . Jahrhunderts ist die in Europa verbreitetste Form des Populismus der sogenannte ,Rechtspopulismus', welcher politisch gesehen darauf aus ist, Macht in Form von Anfeindungen gegenüber anderen, wie etwa ,Fremden' oder ,Ausländern' zu generieren.

\section{Rechtsextremismus:}

Jeder Populismus, der gegen ein ethnisch und/oder national und/oder religiös bestimmtes ,Anderes' gerichtet ist, kann als rechtsextrem angesehen werden. Rechtsextremismus orientiert sich an der ethnischen Zugehörigkeit, an der Infragestellung der rechtlichen Gleichheit der Menschen sowie an einem antidemokratischen, autoritären und antipluralistisch geprägten Gesellschaftsverständnis. Außerdem vertritt Rechtsextremismus die Vorstellung einer ethnisch-kulturell einheitlichen, Volksgemeinschaft'.

Quellen:

Bundeszentrale für politische Bildung: Stichwort Rechtsextremismus. In: Rechtsextremismus. Glossar. Unter: http://www.bpb.de/politik/extremismus/ rechtsextremismus/236157/was-ist-rechtsextremismus, Zugriff: 12.12.2017.

Gold, Johannes (2013): Der Diskurs über Migration in Politik und Medien im Verlauf der österreichischen Zweiten Republik, Dissertation, Universität Wien. Wikipedia, Stichwort Rechtsextremismus. Unter: https://de.wikipedia.org/wiki/Rechtsextremismus, Zugriff: 12.12.2017

\section{Auszug zu Menschenrechten:}

Ihre Grundbotschaft lautet: Allen Menschen kommt kraft ihres Menschseins Würde zu und die Achtung dieser Würde bildet die zentrale Richtschnur für das politische und private Handeln. Wirtschaftliche, soziale und kulturelle Rechte zur Befriedigung der Grundbedürfnisse (medizinische Versorgung, Bildung, Arbeit und soziale Sicherheit) stellen ebenso Grundvoraussetzungen für ein menschenwürdiges Leben dar wie Freiheitsrechte oder die Achtung des Privatund Familienlebens. Menschenrechte sind übergeordnete, den Gesetzen vorgelagerte und jedem Menschen angeborene Rechte, sind aber in vielen Ländern, darunter Österreich, nicht vollständig verbrieft oder umgesetzt.

\section{Existenzielle und die Menschenwürde gestaltende Rechte:}

- Freiheitsrechte (Meinungs-, Gewissens-, Religions-, Vereins- oder Versammlungsfreiheit)

- Gleichheitsrechte (Gleichheit vor dem und durch das Gesetz, Schutz gegen Diskriminierung aufgrund von Geschlecht, Hautfarbe, Religion, ethnischer oder sozialer Herkunft etc.)

- Politische Rechte (Wahlrecht, gleiche Ämterzugänglichkeit, Parteienfreiheit, Petitionsrecht etc.)

- Kollektive Rechte (Selbstbestimmungsrecht der Völker, Schutz von Minderheiten und indigenen Völkern, Recht auf Entwicklung etc.)

- Rechte des Wirtschaftslebens (Eigentum, Freizügigkeit, Erwerbs-, Niederlassungs- oder Dienstleistungsfreiheit)

- Verfahrensrechte (insbesondere im Strafprozess)

- spezifische Rechte für Kinder, Frauen, alte Menschen, Kranke, Behinderte, Migrant/innen, Asylsuchende und sonstige benachteiligte Personengruppen.

Quellen:

Zentrum Polis, Politik Lernen in der Schule (2007): Menschenrechte machen Schule. Unter: http://www.politik-lernen.at/dl/KplpJMJKollMkJqx4KJK/ pa_mrb_10_07_web_09.pdf, Zugriff: 20.12.2017).

Universal Declaration of Human Rights 1948, Art.1.

6 Materialen wurden nach der Durchführung geringfügig überarbeitet. Es wurden sprachliche Vereinfachungen vorgenommen. 


\section{- Methodenblatt Zuordnung der Überschriften zu Kategorien, Themen \& Prinzipien}

AA3: Ordne gemeinsam mit deiner Partnerin/deinem Partner die angeführten Beispiele den sprachlichen Kategorien/Themen / Prinzipien zu (siehe Tabellen 1-3).

Tab. 1: ,Sprachliche Kategorien“ nach Zapata-Barrero \& Rubio-Carbonero 2014

Beispiele: 1. Flüchtlingskrise: Waidhofen zieht nach zwei Jahren Bilanz (Meinbezirk.at, 19.10.2017) 2. Migranten als "Schädlinge" für das tschechische Sozialsystem (science orf.at, 2.9.2015), 3. Türkei droht Europa: „Flüchtlinge werden Europa überschwemmen und übernehmen" (Online Focus, 25.11.2016), 4. Innere Sicherheit: Ein Plan gegen junge, zornige Fremde (Die Presse, 12.5.2016), 5. Bundespräsident Gauck für Grenzschließung - "Das Boot ist voll” (Bundesdeutsche Zeitung, 5.2.2016), 6. 6000 Euro: Immer mehr Migranten kommen per Jacht (Kronen Zeitung, 19. 10.2017), 7. «Ausländer sind kriminell» (Neue Züricher Zeitung, 8.9.2014), 8. Schock-Prognose: Schweden wird bis 2030 Entwicklungsland sein: nur mehr Sozialhilfeempfänger - schlechte Bildung - Ex-Finanzminister: Schweden soll wie Afrika werden (Wochenblick, 23.10.2017)

\begin{tabular}{|c|c|c|}
\hline sprachliche Kategorien & Erklärung & Beispiel(e) \\
\hline $\begin{array}{l}\text { Verwendung von negativen } \\
\text { Verallgemeinerungen }\end{array}$ & $\begin{array}{l}\text { Ein negatives Verhalten/eine negative } \\
\text { Einstellung ist für eine gesamte gesell- } \\
\text { schaftliche Gruppe typisch. Es ist auch eine } \\
\text { Art Vereinfachung, die Ausnahmen nicht } \\
\text { berücksichtigt. Beide sind die } \\
\text {,Basis' um Vorurteile zu erzeugen. } \\
\text { Beispiel: Alle Studierenden sind faul. }\end{array}$ & \\
\hline $\begin{array}{l}\text { Verwendung von negativen } \\
\text { Übertreibungen (=Hyperbeln) }\end{array}$ & $\begin{array}{l}\text { Negative Verallgemeinerungen werden } \\
\text { dramatisiert. } \\
\text { Beispiel: Arbeitslose, die nichts arbeiten wol- } \\
\text { len, gibt es bei uns wie Sand am Meer. }\end{array}$ & \\
\hline $\begin{array}{l}\text { Verwendung von negativen } \\
\text { Sinnbildern (=Metaphern) }\end{array}$ & $\begin{array}{l}\text { Man spricht von einer Metapher, wenn } \\
\text { ein Satz / ein Wort nicht wörtlich gemeint } \\
\text { ist, sondern im übertragenen Sinne - also } \\
\text { bildhaft. } \\
\text { Funktion: Übertreibung \& Dramatisierung } \\
\text { Folge: kann Angst und Ablehnung erzeugen } \\
\text { Beispiel: Ein Islamisierungstsunami wird } \\
\text { erwartet. }\end{array}$ & \\
\hline $\begin{array}{l}\text { Verwendung entmenschlichen- } \\
\text { der Begriffe }\end{array}$ & $\begin{array}{l}\text { Menschen werden als Tiere oder Dinge } \\
\text { dargestellt und somit sozusagen, ent- } \\
\text { menschlicht'. } \\
\text { Beispiel: Sozialschwache Menschen sind } \\
\text { Schmarotzer und Parasiten unserer Gesell- } \\
\text { schaft. }\end{array}$ & \\
\hline
\end{tabular}


Tab. 2: Themen \& strategischer Einsatz dieser nach Zapata-Barrero \& Rubio-Carbonero 2014

Beispiele: 1. Städte fürchten Sozial-Kollaps wegen EU-Ausländern (WeltN24, 25.1.2016) 2. „Die Gewalt und Kriminalität der ,Flüchtlinge' explodiert - doch wen interessiert's" (SaaleJournal, 20.3.2017), 3. "Beitrag übererfüllt": Österreich will keine Flüchtlinge aus EU-Programm aufnehmen (Die Presse, 27.3.2017), 4. 1.000,- Kindergeld! Warum bekommen AsylantenKinder das 5-fache des deutschen Kindergeldes? (gleichberechtigt.eu, 15.11.2016), 5. Arbeitsmarkt: Österreich leidet unter der Zuwanderung aus Osteuropa (Profil, 20.2.2016), 6. Trump: Muslimische Zuwanderung zerstört Europa (Berlin Journal, 5.5.2016)

\begin{tabular}{|l|l|l|}
\hline $\begin{array}{l}\text { Themen (+ strategischer } \\
\text { Einsatz) }\end{array}$ & Erklärung & Beispiel(e) \\
\hline Thema der Bedrohung & $\begin{array}{l}\text { Absolute ,Wahrheiten', die aus der Gesell- } \\
\text { schaft hervorgehen und auch nicht hinter- } \\
\text { fragt werden. } \\
\text { Beispiel: Online Handel bedroht österreichi- } \\
\text { sche Unternehmen. }\end{array}$ & \\
\hline Thema der Belastung & $\begin{array}{l}\text { siehe oben } \\
\text { Beispiel: Die, Alten' liegen den, Jüngeren' } \\
\text { auf der Tasche. }\end{array}$ & \\
\hline Thema der Bevorteilung & siehe oben \\
Beispiel: Superreiche weiterhin steuerfrei!
\end{tabular}

Tab. 3: Prinzipien zur Bestimmung von Rechtsextremismus nach Holzer 1994

Beispiele: 1. Erstmals mehr als eine Million Nichtösterreicher in Österreich (Der Standard, 19.02.2013) 2. Statistik: Neue Jobs gehen an Ausländer (Die Presse, 09.04.2016), 3. Integrationsgesetz Wertekurse ausgebaut - Burkaverbot endgültig fix (Kronen Zeitung, 28.03.2017), 4. Höhere Asylkosten. Muslime mit Zweitfrau: Bis zu $3000 €$ Sozialhilfe (Kronen Zeitung, 02.02.2016), 5. Was den Islam gefährlich macht (somit auch seine Anhänger): Eine Religion auf Leben und Tod (Profil, 12.01.2015)

\begin{tabular}{|c|c|c|}
\hline $\begin{array}{l}\text { Prinzipien zur Bestimmung von } \\
\text { Rechtsextremismus }\end{array}$ & Erklärung & Beispiel(e) \\
\hline Prinzip der Natur / Natürlichkeit & $\begin{array}{l}\text { Alles Abgelehnte wird als widernatürlich } \\
\text { bezeichnet und somit entwertet. } \\
\text { Beispiel: Homosexualität ist unnatürlich. }\end{array}$ & \\
\hline $\begin{array}{l}\text { Prinzip der unveränderbaren } \\
\text { Charaktermerkmale eines Vol- } \\
\text { kes/einer Volksgemeinschaft }\end{array}$ & $\begin{array}{l}\text { Die Ansicht, dass ein Volk mit, unverän- } \\
\text { derbaren Eigenschaften' ausgestattet ist. } \\
\text { Die Eigenschaften einer Einzelperson sind } \\
\text { weniger wichtig. } \\
\text { Beispiel: Es hat keinen Sinn, von Österrei- } \\
\text { chern Pünktlichkeit zu erwarten. }\end{array}$ & \\
\hline $\begin{array}{l}\text { Prinzip ,Ethnozentrismus und } \\
\text {-pluralismus' }\end{array}$ & $\begin{array}{l}\text {,Deutschland den Deutschen, die Türkei } \\
\text { den Türken' usw. Es verfolgt eine starke } \\
\text { Integrations- und Ausgrenzungsfunktion. } \\
\text { Beispiel: Bären siedeln sich wieder in Öster- } \\
\text { reich an. (Tierhabitate, etc.) }\end{array}$ & \\
\hline Prinzip ,Sündenbockfunktion' & $\begin{array}{l}\text { Gruppen, wie etwa, die Fremden', wird die } \\
\text { (alleinige) Verantwortung für beispielsweise } \\
\text { soziale Benachteiligungen und Missstände } \\
\text { zugeschrieben. Der einfachste Weg ist, } \\
\text { eigene Fehler auf feindliche Gruppen zu } \\
\text { projizieren. } \\
\text { Beispiel: Die junge Generation ist schuld } \\
\text { daran, dass das österreichische Sozialsys- } \\
\text { tem nicht mehr funktioniert - Studien, etc. } \\
\text { verhindern Zahlungen. }\end{array}$ & \\
\hline
\end{tabular}


- Identifizierung von Rechtsextremismus in Onlinemedien (Gruppenarbeit) Beispiel Onlineartikel mit Kommentarfunktion

AA4/1: Bearbeitet gemeinsam folgenden Artikel bzw. die Kommentare (Link: http://www.krone.at/601526)

1. Institutioneller Rahmen/Formalia zum Medium: Ermittelt folgende Informationen bezogen auf die Kommentare (siehe am Ende des Artikels):

- Um welches Medium handelt es sich?

- Wer hat die Beiträge verfasst?

- An wen sind die Beiträge adressiert?

- Welche Akteurinnen und Akteure sind beteiligt?

- Wer kommt innerhalb der Beiträge zu Wort?

2. Inhaltliche Ebene: Skizziert, wie sich die Kommentare zum Inhalt des Artikels verhalten? Bedienen sich die Kommentare der Leser/innen ähnlicher Argumentationslinien?

3. Fokus sprachliche Kategorien/Themen/Prinzipien: Skizziert, wie sich die Kommentare zum Inhalt des Artikels verhalten? Bedienen sich die Kommentare der Leser/innen ähnlicher Argumentationslinien?

- Welche sprachlichen Kategorien/Themen/Prinzipien werden im Artikel bzw. in den Kommentaren verwendet? Bezieht euch dabei auf die Tabellen 1-3 (Mindestanalyse $=5$ Kommentare).

- Werden bestimmte/auffällige Ausdrücke öfter verwendet? Wenn ja, welche Intentionen könnten dahinterstecken?

4. Reflexionsaufgaben: Bezieht Stellung. Wie wirken die Kommentare auf euch? Diskutiert, ob die Menschenrechte geachtet werden (siehe Methodenblatt Auszug Menschenrechte).

Zusatzaufgabe: Wird der Text mit einer Bebilderung unterstützt? Wenn ja, dann beschreibt diese zunächst so objektiv als möglich und interpretiert sie anschließend. 
Beispiel Einzelfallkarte mit dem Fokus auf Salzburg

AA4/2: Bearbeitet gemeinsam folgende Karte bzw. deren Einträge

(Link: https://www.google.com/maps/d/viewer?mid=1_rNT3k2ZXB-f9z-2nSFMIBQKXCs\&hl=en_US\&ll=48.220787 $34798839 \% 2 \mathrm{C} 16.38005995000003 \& z=11)^{7}$

1. Institutioneller Rahmen/Formalia zum Medium: Ermittelt folgende Informationen bezogen auf die Einzelfallkarte:

- Um welches Medium handelt es sich?

- Wer hat die Beiträge verfasst?

- An wen sind die Beiträge adressiert?

- Welche Akteurinnen und Akteure sind beteiligt?

- Wer kommt innerhalb der Beiträge zu Wort?

2. Inhaltliche Ebene: Erläutert, auf welche Informationen sich die von euch analysierten Einträge stützen und skizziert auch, wie sich die Überschriften der Einträge zum Inhalt der dahinterliegenden Beiträge verhalten?

3. Fokus sprachliche Kategorien/Themen/Prinzipien: Skizziert, wie sich die Kommentare zum Inhalt des Artikels verhalten? Bedienen sich die Kommentare der Leser/innen ähnlicher Argumentationslinien?

- Welche sprachlichen Kategorien/Themen/Prinzipien werden in den Einträgen verwendet? Bezieht euch dabei auf die Tabellen 1-3 (Mindestanalyse = 5 Einträge).

- Werden bestimmte/auffällige Ausdrücke öfter verwendet? Wenn ja, welche Intentionen könnten dahinterstecken?

4. Reflexionsaufgaben: Bezieht Stellung. Wie wirken die Einträge auf euch? Diskutiert, ob die Menschenrechte geachtet werden (siehe Methodenblatt Auszug Menschenrechte).

Zusatzaufgabe: Wie wirkt das "Startbild" der Einzelfallkarte auf euch? Interpretiert es gemeinsam. https://www.google.com/maps/d/viewer?mid=1_rNT3k2ZXB-f9z-2nSFMIBQKXCs\&hl=en_US\&ll=51.3611944393 $14694 \% 2 C 6.969721633496192 \& z=5)$.

Die Einzelfallkarte wurde zu Beginn des Jahres 2018 vom Netz genommen und ist nicht mehr zugänglich. Allerdings ist damit zu rechnen, dass weitere Karten, die ähnliche Ziele verfolgen, wieder erstellt werden. 


\section{Durchführung der Unterrichtseinheiten}

Wie bereits im Planungsraster ersichtlich, handelte es sich zunächst um eine Doppeleinheit zu jeweils fünfzig Minuten. Zwischen den beiden Einheiten war eine fünfminütige Pause vorgesehen, die kurzerhand zur Gruppeneinteilung genutzt wurde. Zwischen den ersten beiden und der dritten Unterrichtseinheit, in der ein zwanzig minütiges Fazit stattfand, verstrichen ferienbedingt drei Wochen.

Die Unterrichtseinheiten wurden mittels Teamteaching durch die Autorin und den Autor im Rahmen des GW-Unterrichts einer vierten Klasse HTL am Holztechnikum Kuchl durchgeführt. Die Klasse wies eine Anzahl von 30 Schülerinnen und Schülern (28 Jungen und zwei Mädchen) auf und wurde im Fach Geographie und Wirtschaftskunde von Frau Mag. Renate Ager unterrichtet. Die Klasse beschäftigte sich in den vorherigen Unterrichtseinheiten mit der Europäischen Union. Thematisch konnte gut angeknüpft werden, da die Europäische Union einen ge- schaffenen Raum für Identitäten darstellt, dessen Ziel es ist, sich gegenüber etwas ,Anderem '/,Fremdem abzugrenzen (vgl. Drüeke 2001).

\subsection{Ergebnisse der Schüler/innen und Erfahrungen}

Assoziationen. In einer ersten Annäherung an den Lernerfolg der Unterrichtsumgebung erhoben wir vor und nach Durchführung der Unterrichtsumgebung die Assoziationen der Schüler/ innen. Diese wurden in der Folge gesammelt und in eine Word Cloud umgewandelt (siehe Abb. 1).

Anhand der beiden Abbildungen kann gezeigt werden, dass der Unterricht zu einer deutlichen Verbreiterung der Assoziationen und damit zu einer kritischeren Betrachtung des Konzept Rechtsextremismus geführt hat. Neben einer reinen Verbreiterung der Assoziationen können auch Verschiebungen in den Gewichten festzustellen sein. Beide Prozesse sind teilweise

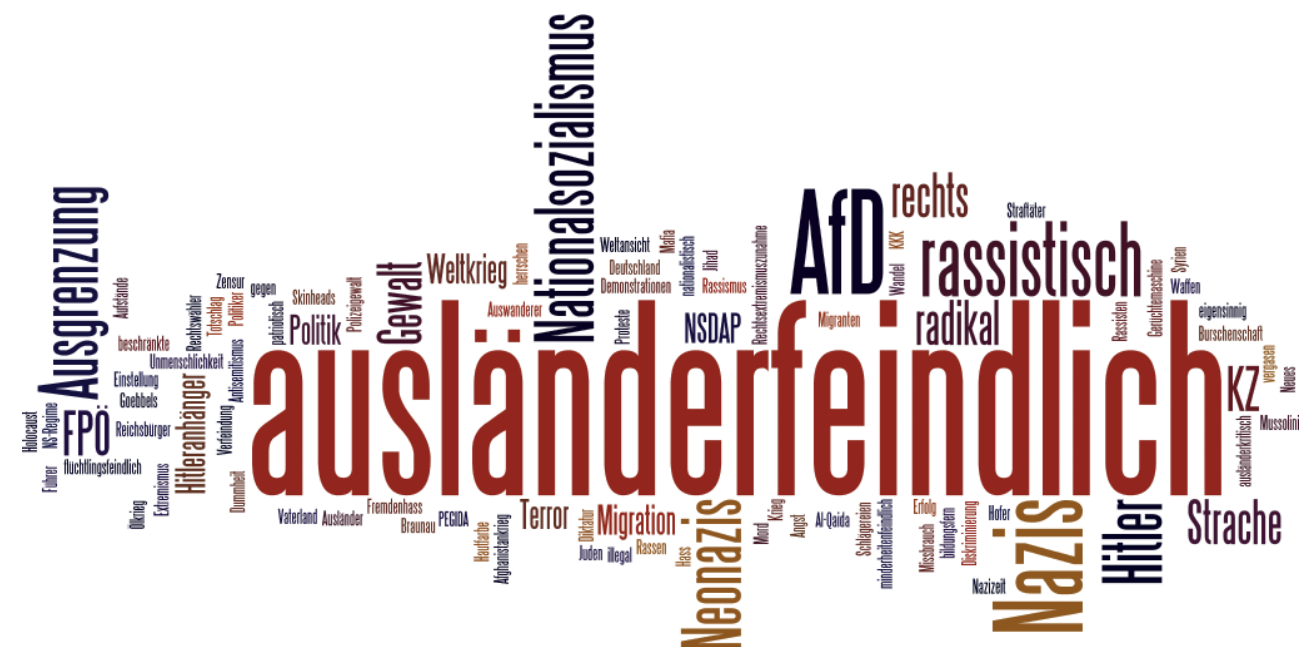

Prä-Assoziationen

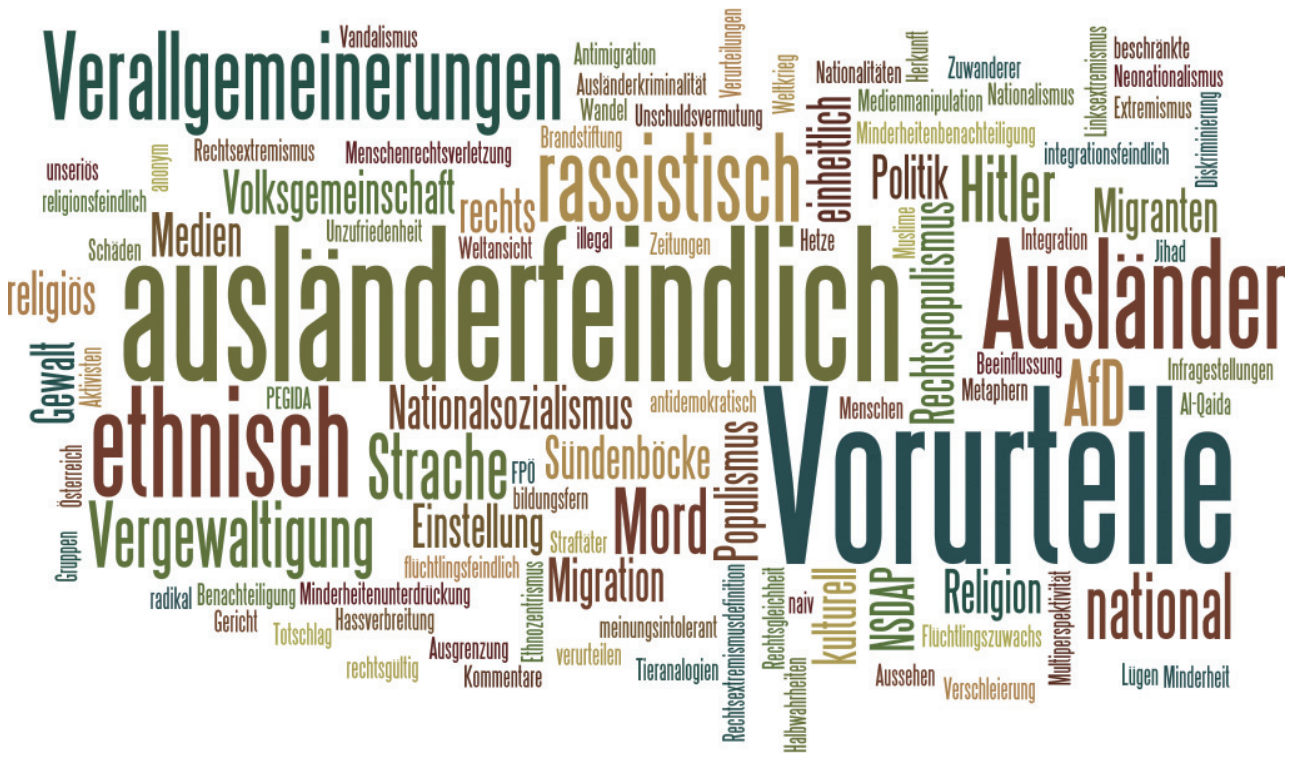

Post-Assoziationen

Abb. 1: Prä- und Post-Assoziationen der Schüler/innen. Freie Assoziation, Mehrfachnennungen möglich. 
direkt zu den verwendeten Materialien zu relativieren.

Trennschärfe Rechtspopulismus / Rechtsextremismus in (neuen) Medien: Die Schüler/innen waren sich schnell einig, dass die Grenzen zwischen Rechtspopulismus und Rechtsextremismus verschwimmen und daher eine scharfe Abgrenzung unmöglich erscheint.

Zuordnung der Überschriften zu Kategorien, Themen \& Prinzipien: Die Schüler/innen zeigten zu Beginn der Übung deutliche Unsicherheiten im Bereich des sozialwissenschaftlichen Arbeitens. Sie konnten zunächst nur schwer damit umgehen, keine eindeutigen Lö- sungen $\mathrm{zu}$ erhalten und somit ihre subjektiven Betrachtungsweisen gegenüber den Partner/innen zu argumentieren. Wir betrachten das Aufzeigen dieser Unsicherheit der Zuordnung / Codierung als wesentlich für den Lernprozess der Schüler/innen.

Medienanalyse: Eine Gruppe widmete sich einem Online-Artikel der ,Krone' mit dem Titel ,Kritik am Islam. Leben mit Zuwanderern: Stimmung verschlechtert'. Zudem bietet der Online-Artikel eine Kommentarfunktion, die von zahlreichen Leserinnen und Lesern genutzt wurde. Folgend finden sich sowohl die gestellten Aufgaben als auch die konkreten Antworten der Schüler/innen.

\section{AA4/1: Bearbeitet gemeinsam folgenden Artikel bzw. die Kommentare}

(Link: http://www.krone.at/601526)

1. Institutioneller Rahmen

\section{Formalia zum Medium}

- Um welches Medium handelt es sich?

- Wer hat die Beiträge verfasst?

- An wen sind die Beiträge adressiert?

- Welche Akteurinnen und Akteure sind beteiligt?

- Wer kommt innerhalb der Beiträge zu Wort?

\author{
Ergebnisse der Schüler/innen \\ Boulevardzeitung \\ anonyme Verfasser \\ an Österreicher und Ausländer, \\ an Politiker \\ Meinungsforscher Peter Hajek \\ ÖIF-Geschäftsführer \\ Muslime \\ Befragte \\ Menschen, die kommentieren
}

nur der Autor

2. Inhaltliche Ebene: Skizziert, wie sich die Kommentare zum Inhalt des Artikels verhalten? Bedienen sich die Kommentare der Leser/innen ähnlicher Argumentationslinien? Die Schüler/innen identifizierten folgende Merkmale:

- die Kommentare gehen wenig auf den Artikel ein, sondern verarbeiten eigene Erfahrungen oder stützen sich auf allgemeine Vorurteile

- $\quad$ in den Kommentaren findet man eine aggressive Sprache gegenüber Ausländern

- die meisten Kommentare sind weniger sachlich und haben keinen Bezug zu einer wissenschaftlichen Quelle/im Gegensatz zum Artikel: hier erfolgt der Verweis auf den Meinungsforscher Peter Hajek

- oftmals wird nur geschrieben, dass Ausländer nichts in Österreich zu suchen haben, aber Begründungen dafür fehlen

- Erfahrungen von verschiedenen Personen werden geteilt, bei denen ,Asylanten' scheinbar bevorzugt wurden

Die Schüler/innen sind in der Lage, die Strukturen sowie die stark vereinfachenden Aussagen zu erkennen. Zudem konnten sie feststellen, dass die analysierten Kommentare einen wenig sachlichen Charakter aufweisen, die dahinterliegenden Argumentationslinien meist sehr dünn sind und auf Vorurteilen basieren.

\section{Fokus sprachliche Kategorien/Themen/Prinzipien:}

- Welche sprachlichen Kategorien/Themen/Prinzipien werden im Artikel bzw. in den Kommentaren verwendet? Bezieht euch dabei auf die Tabellen 1-3 (Mindestanalyse $=5$ Kommentare).

- Werden bestimmte / auffällige Ausdrücke öfter verwendet? Wenn ja, welche Intentionen könnten dahinterstecken? 
Man kann nur mehr hoffen, dass die neue Regierung hier ein paar scharfe Einschnitte in Bezug auf die diversen Geldleistung macht. Und das ziemlich prompt. Und wenn sie dann einen Wirbel machen, ab in die Heimat mit der Hercules. Wir wollen unser Österreich wieder zurück.

\section{Thema der Belastung, Prinzip Sündenbockfunktion}

Der Saat geht auf!

negative Übertreibung; Thema der Bedrohung, Prinzip Ethnozentrismus, Metapher

Nochmals: Der Islam hat in Europa und im speziellen in Österreich NICHTS verloren. Im Prinzip müßte er schon lange verboten sein weil er eine Säkularität unmöglich macht. Das die Fehlentscheidungen unserer Politiker blutig werden steht zweifellos fest. Danke \& Freundschaft!

Prinzip Ethnozentrismus, Thema der Bedrohung

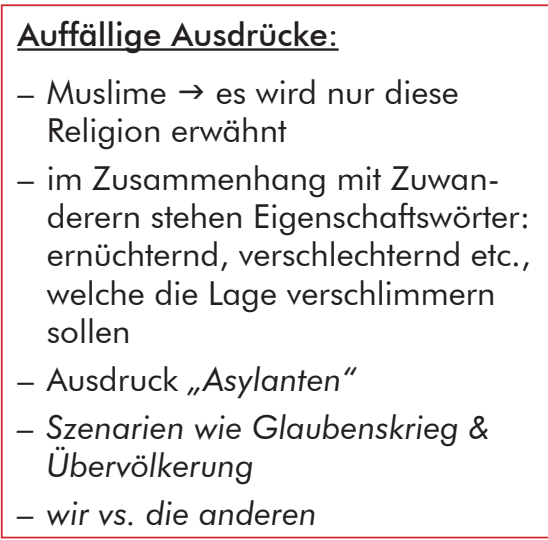

Abb. 2: Antworten der Schüler/innen: Semantische Kategorien, Topoi \& Prinzipien des Rechtsextremismus. Aufbereitung für die Nachbesprechung: Karin Golser

Den Schülerinnen und Schülern gelingt die Herausarbeitung von semantischen Kategorien, Topoi sowie Prinzipien und sie können somit das erworbene Handwerkszeug (siehe Zuordnungsübung AA3) reflektiert anwenden. Zudem stellen sie durch Diskussionen in den Gruppen fest, dass Mehrfachzuordnungen möglich sind.

4. Reflexionsaufgaben: Bezieht Stellung. Wie wirken die Kommentare auf euch? Diskutiert, ob die Menschrechte geachtet werden (siehe Methodenblatt Auszug Menschenrechte).

Subjektive Schüler/innenaussagen zu diesen Fragen waren in der gegenständlichen Unterrichtsdurchführung folgende:

- die Kommentare wirken hauptsächlich negativ behaftet und sind aggressiv

- die Leute waren vermutlich wirklich wütend oder genervt, als sie die Kommentare geschrieben haben

- wenige Menschen versuchen objektiv zu kommentieren

- es kommt uns so vor, als dass die Leute einfach schreiben, was sie hören und selbst keine Ahnung davon haben

- die Kommentare sind teilweise so geschrieben, als ob die Verfasser selbst keine Ahnung von der deutschen Sprache / Rechtschreibung hätten

- nein, es wird kaum bis gar nicht auf die Menschenrechte geachtet: die Kommentare sind hauptsächlich voll mit Wut und die Leute posten ohne sich ein "Blatt vor den Mund zu nehmen"

- die Meinungen der Menschen sind oft negativ behaftet, es scheint als ob sie wirklich gar nichts Positives sehen!

Die von den Schülerinnen und Schülern konstatierte negative Grundhaltung der Kommentare trifft gleichzeitig auch auf die Haltung der Schüler/innen den Kommentaren gegenüber zu. Inwieweit dies aus der Wahrnehmung der Erwartungshaltungen der Durchführenden resultiert, oder aus dem Unterrichtsdesign - das sich aber auf wesentliche Dokumente der Demokratie und der Menschenrechte bezieht - kann nicht festgestellt werden. Wir halten dies aber für zulässig. Die Schüler/innen beantworten die Frage, die auf die Wirkung der Kommentare abzielt, äußerst umfangreich. Weiter kommen sie zum Schluss, dass viele Kommentare den Menschenrechten widersprechen, allerdings fallen ihre Begründungen eher spärlich aus.

\subsection{Schwierigkeiten}

Auffallend war, dass sich Gruppen, die Zeitungsartikel als Recherchegrundlage hatten, um einiges leichter taten als jene, die auf Basis der Einzelfallkarte arbeiteten. Um den Arbeitsauftrag entsprechend lösen zu können, sollten die Schüler/innen daher bereits über ein gewisses Maß an Kartenlesekompetenz verfügen, sprich sich des konstruierten Charakters von Karten bewusst sein. Denn es gilt verborgene und verschwiegene Informationen sowie eingearbeitete Interessen, Werte und Normen sichtbar werden zu lassen 
(vgl. Gryl 2009). Eine umfangreichere und präzisere schriftliche Arbeitsanleitung zur Beschreibung der Karte hätte möglicherweise Unklarheiten abwenden können. An dieser Stelle kann auf das Modell der konstruktivistischen Kartenlesekompetenz von Inga Gryl (2009) verwiesen werden. Die Durchführung der Unterrichtsumgebung zeigte, dass für ein besseres Verständnis der Einzelfallkarte eine adäquate Anleitung im Vorhinein vorgenommen werden sollte. Dafür würde sich beispielsweise ein Kurzverweis auf Gryls entworfene, Werkzeugkiste', die zur Dekonstruktion von Karten benötigt wird, eignen.

Neben eigens gesammelten Erfahrungen bekamen wir ein ausführliches und wertvolles Feedback durch die GW-Lehrerin der Klasse, die sich vor allem zu Beginn der Unterrichtseinheiten eine Präsentation der Stundenstrukturierung und der konkreten Vorgehensweise wünschen würde, sodass auch die Schüler/innen auf das gemeinsame Vorhaben vorbereitet werden. Aber gerade was die Absichten bzw. die Ziele des von uns konzipierten Unterrichts betrifft, sollte wenig verraten werden, sodass die Reflexionen der Schüler/innen nicht aufgrund der zuvor genannten Zielsetzung verfälscht würden. Wertvolles Feedback erhielten wir auch zum sprachlichen Niveau des Methodenblattes ,Rechtspopulismus/Rechtsextremismus in (neuen) Medien'. Sie sind entsprechend in der vorliegenden Version von AA2 angepasst und vereinfacht enthalten. Zudem hätte man Zusatzaufgaben, die bei unserem Entwurf erst für die Gruppenarbeiten vorlagen, anführen können, um die verschiedenen Bearbeitungsniveaus ausgleichen zu können. Eine weitere Anmerkung bekamen wir zur Gruppengröße, die durchaus angenehm schien, aber ein Gerät pro Gruppe sei zu wenig gewesen. Als wir die Einheit konzipierten, war es uns ein großes Anliegen, dass der Austausch innerhalb der Gruppen im Fokus steht. Daher sahen wir zwei Rechner eher als Gefahr an, sodass in Untergruppen oder gar einzeln gearbeitet wird. Als Vorbild für eine erneute Durchführung könnte man jene Gruppe heranziehen, die zwar auf zwei Rechnern, aber in einem Onlinedokument vernetzt, arbeitete.

\section{Fazit}

Die vorliegende Unterrichtskonzeption für die Sekundarstufe II wurde unseres Erachtens erfolgreich mit 17/18-jährigen Schülerinnen und Schülern durchgeführt und die Materialien entsprechend angepasst. Wir sind der Meinung, dass sich auf Basis dieses forschenden Zugangs sowohl das Interesse der Schüler/ innen wecken lässt als auch die Assoziationsdichte zu Phänomenen des Rechtsextremismus deutlich steigert. Eine Durchführung mit jüngeren Schülerinnen und Schülern steht noch aus und könnte aufgrund der Komplexität von Thema und Definitionen zu Problemen führen. Naturgemäß sind die Materialien auf jeweils aktuelle Artikel und Medien übertragbar.

Klar wurde allerdings auch, dass die Analyse sozialer Medien dort noch nicht ausgereizt ist, wo nicht nur Text, sondern Karten und Bilder in die Analyse einfließen. Hier erscheinen erweiterte Vorbereitungen der Schüler/innen als notwendig, die auf die Veränderungen der Kartographie zu einem Laienmedium aufmerksam machen. Gleichzeitig wäre es durchaus spannend, eine ähnliche Unterrichtsstruktur auf Basis geeigneter semantischer Kategorien und Topoi auch für linksextreme Bewegungen zu entwickeln.

\section{Dank}

Die Autorin und der Autor bedanken sich bei der Schulleitung des Holztechnikum Kuchl für die Möglichkeit der Durchführung des Unterrichtsprojektes; beim OpenAccess-Fonds der Universität Salzburg für die Unterstützung der Veröffentlichung, sowie bei zwei anonymen Reviewern für ihrehilfreichen Kommentare.

\section{$7 \quad$ Literatur}

Bailer-Galanda, B. (o. J.): Zum Begriff des Rechtsextremismus. https://www.doew.at/erkennen/rechtsextremismus/ rechtsextreme-organisationen/zum-begriff-des-rechtsextremismus (22.2.2018).

BMB - Bundesministerium für Bildung (2016): Lehrplan der AHS-Oberstufe für Geographie und Wirtschaftskunde. In: Bundesgesetzblatt Nr. 219 v. 19.8.2016, S. 59-67. https:// www.ris.bka.gv.at/Dokumente/BgblAuth/BGBLA_2016_ II_219/BGBLA_2016_II_219.pdf

BMBWF - Bundesministerium für Bildung, Wirtschaft und Forschung (2015): Unterrichtsprinzip Politische Bildung, Unter: https://bildung.bmbwf.gv.at/schulen/ unterricht/prinz/politische_bildung.html (26.2.2018).

BMJ - Bundesministerium für Justiz (2017): Erlass vom 17. September 2017 zum Leitfaden zum Tatbestand der Verhetzung (\$ 283 StGB), https://www.ris.bka.gv.at/ Dokumente/Erlaesse/ERL_07_000_20170917_BMJ_ S215_001_0002_IV_1_2017/ERL_07_000_20170917_ BMJ_S215_001_0002_IV_1_2017.pdf (25.6.2018).

Bundeszentrale für politische Bildung (o.J.): Stichwort Rechtsextremismus. In: Rechtsextremismus. Glossar. http://www.bpb.de/politik/extremismus/rechtsextremismus/236157/was-ist-rechtsextremismus (26.2.2018).

Drüeke, R. (2011): Politische Kommunikationsräume im Internet. Überlegungen zu Raum und Öffentlichkeit im Kontext der Migrationsdebatte um Arigona Zogaj, Dissertation, Universität Salzburg. 
Fridrich, Ch. (2016): Basiskonzepte in Geographie und Wirtschaftskunde - ein Vorschlag für die Sekundarstufe I. In: GEOGRAZ 59, 24-31.

Fuchs, C. (2016): Racism, Nationalism and Right-Wing Extremism Online: The Austrian Presidential Election 2016 on Facebook. In: Momentum-Quarterly - Zeitschrift für Sozialen Fortschritt 5/4, 200-264.

Gold, J. (2013): Der Diskurs über Migration in Politik und Medien im Verlauf der österreichischen Zweiten Republik, Dissertation, Universität Wien.

Golser, K. \& T. Jekel (2017): What is spatial about rightwing discourses? In: GI_Forum 5/2, 36-48.

Gryl, I. (2009): Kartenlesekompetenz. Ein Beitrag zum konstruktivistischen Geographieunterricht (= Materialien zur Didaktik der Geographie- und Wirtschaftskunde 22). Wien.

Gulis, W. (2013): Migrationsdiskurs in Österreich. Entstehung eines Migrationsdiskurses in der zweiten Republik Österreichs, Saarbrücken.

Holzer, W. (1994): Rechtsextremismus. Konturen, Definitionsmerkmale und Erklärungsansätze. In: Dokumentationsarchiv des österreichischen Widerstandes (Hrsg.): Handbuch des österreichischen Rechtsextremismus. Wien: Deutike, 12-96.

van Houtum, H. \& T. van Naerssen (2002): Bordering, Ordering and Othering. In: Tjidschrift voor Economische en Sociale Geografie 93/2, 125-136.

Jaschke, H.-G. (1991): Streitbare Demokratie und innere Sicherheit. Opladen: Westdeutscher Verlag.

Jaschke, H.-G. (1994): Rechtsextremismus und Fremdenfeindlichkeit. Opladen: Westdeutscher Verlag.

Jekel, T. \& F. Huber (2005): Barockes Salzburg: Zur Normalisierung von Raumkonstruktionen. In: Flitner, M. \& Lossau, J.: Themenorte. Produktion und Durchsetzung von Raumbildern. Münster: Lit, 91-106.

Jekel, T., M. Lehner \& R. Vogler (2017): Mapping the Far Right: Geomedia in an Educational Response to Right-Wing Extremism. In: International Journal of Geoinformation, 6/294, 1-14. https://doi.org/10.3390/ ijgi6100294.
Jekel, T. \& H. Pichler (2017): Vom GW-Unterrichten zum Unterrichten mit geographischen und ökonomischen Konzepten. $\mathrm{Zu}$ den neuen Basiskonzepten im österreichischen GW-Lehrplan AHS Sek II. In: GW-Unterricht, $147,5-15$.

Mernyi, W. \& C. Bauer (2017): Rechtsextrem. Symbole-CodesMusik-Gesetze-Organisationen, Wien: ÖGB-Verlag.

Meyer, H. (2011): Unterrichtsmethoden 2. Praxisband. Berlin: Cornelsen.

Pelinka, A. (2013): Right-Wing Populism: concept and typology. In: Wodak, R., M. KhosraviNik \& B. Mral: Right-Wing Populism in Europe. Politics and Discourse. London, New York, 3-22.

Pichler, H. (2017): Grenzzäune und Grenzmauern - Bildimpulse zu einem kritischen Raumverständnis. Ein Praxisbeispiel zur Implementierung von Basiskonzepten im GW-Unterricht. In: GW-Unterricht 147, 32-41.

Teml, H. \& H. Teml (2006): Erfolgreiche Unterrichtsgestaltung. Wege zu einer persönlichen Didaktik. Innsbruck: Studienverlag.

United Nations (1948): Universal Declaration of Human Rights Art. 1. Unter: http://www.un.org/en/universaldeclaration-human-rights/index.html (23.6.2018).

Vielhaber, Ch. (1999): Vermittlung und Interesse - Zwei Schlüsselkategorien fachdidaktischer Grundlagen im Geographieunterricht. In: Vielhaber, Ch. (Hrsg.): Fachdidaktik kreuz und quer. Bd. 15 der Materialien zur Didaktik der Geographie u. Wirtschaftskunde.

Wodak, R. (2012): Vorurteil, Rassismus und Diskurs. In: Pelinka, A. (Hrsg.): Vorurteile. Ursprünge, Formen, Bedeutung. Berlin/Boston: Walter de Gruyter.

Zapata-Barrero, R. \& G. Rubio-Carbonero (2014): Monitoring xenophobic political discourses: a pilot study in Catalonia. Barcelona: GRITIM-UPF (= Policy Series 2). https://www.upf.edu/gritim/_pdf/gritimps2.pdf (20.7.2018).

Zentrum Polis, Politik Lernen in der Schule (2007): Menschenrechte machen Schule. http://www.politiklernen.at/dl/KplpJMJKollMkJqx4KJK/pa_mrb_10_07_ web_09.pdf (20.12.2017). 